\title{
Miotomía endoscópica por vía oral (POEM) para el tratamiento de acalasia: nuestras primeras experiencias
}

\section{Peroral endoscopic myotomy (POEM) for treatment of achalasia: our first experiences}

Jesús A. Rodríguez F, ${ }^{1}$ Habib J. Mantilla G., ${ }^{2}$ Luis F. Pineda 0., ${ }^{3}$ Álvaro A. Jalal E., ${ }^{4}$ Héctor J. Escobar C., ${ }^{5}$ Víctor H. Arbeláez M., ${ }^{6}$ Martín A. Gómez Z., ${ }^{7}$ Carlos A. Salgar G., José E. Salgar V. ${ }^{8}$

\footnotetext{
1 Especialista en cirugía gastrointestinal y endoscopia digestiva. Organización Clínica General del Norte, Barranquilla. Clínica Shaio, Bogotá D. C., Colombia.

2 Especialista en cirugía general. Hospital Militar Central, Bogotá D. C., Colombia.

3 Especialista en medicina interna, gastroenterología y epidemiología; profesor de la Universidad Nacional de Colombia, servicio de motilidad gastrointestinal; director científico del Instituto GutMédica. Bogotá D.C., Colombia.

${ }^{4}$ Especialista en cirugía general y endoscopia digestiva. Organización Clínica General del Norte. Barranquilla, Colombia.

5 Cirujano general, Clínica MedSalud. Fusagasugá, Colombia.

${ }^{6}$ Especialista en medicina interna y gastroenterología; profesor de posgrado de la Universidad Nacional de Colombia; servicio de gastroenterología y endosonografía del Instituto GutMédica. Bogotá D. C. Colombia.

Médico internista y gastroenterólogo. Profesor asociado de la Universidad Nacional de Colombia. Gastroenterólogo del Hospital Nacional Universitario. Bogotá D. C., Colombia.

${ }_{8}$ Cirujano general y gastroenterólogo. Clínica San José. Cúcuta, Colombia.

Correspondencia: Jesús A. Rodríguez F.

Correo: doctorjesusrodriguezf@yahoo.com. Institución: Organización Clínica General del Norte. Barranquilla, Colombia.

Fecha recibido: $19-12-17$

Fecha aceptado: 08-03-18
}

\begin{abstract}
Resumen
Introducción: la miotomía endoscópica por vía oral (POEM) se diseñó inicialmente para el manejo de la acalasia, que es un trastorno crónico y progresivo de la motilidad esofágica con hipertensión del esfínter esofágico inferior (EEI), para la cual el manejo estándar, hasta ahora, ha sido la cirugía de Heller; sin embargo, esta nueva técnica ofrece resultados muy comparables y prometedores. Materiales y métodos: se realizó una revisión retrospectiva de las historias clínicas de los pacientes con acalasia operados por POEM en una institución de Colombia por un solo cirujano endoscopista. Resultados: se encontraron 4 pacientes con acalasia, todas con Eckardt $\geq 10$ y presión $\mathrm{EEI} \geq 30 \mathrm{~mm} \mathrm{Hg}$; el tiempo quirúrgico osciló entre 81-120 minutos; en el seguimiento, la reducción en la presión del EEl fue mayor al $50 \%$ prácticamente en todos los pacientes, encontrando valores cercanos a la normalidad y con resultados de Eckardt que demuestran éxito en la terapia POEM a los 2 meses de evaluación. Las complicaciones fueron resueltas en el intraoperatorio, con mortalidad de $0 \%$. Conclusiones: El manejo de la acalasia mediante POEM ha demostrado ser una técnica eficaz y segura. Nuestros primeros hallazgos concuerdan con los resultados descritos en la literatura. Este es el primer paso para la implementación de este novedoso abordaje en el manejo de la acalasia en la población colombiana.
\end{abstract}

\section{Palabras clave}

Acalasia, desórdenes de la motilidad esofágica, miotomía endoscópica por vía oral, miotomía de Heller.

\begin{abstract}
Introduction: Peroral endoscopic myotomies (POEMs) were initially designed to manage achalasia, a chronic and progressive esophageal motility disorder with lower esophageal sphincter hypertension (LES). Until recently, standard treatment had been Heller's surgery, but this new technique offers very promising pressure. Materials and methods: This is a retrospective review of the clinical histories of patients with achalasia who underwent POEM performed by one endoscopic surgeon at a Colombian institution. Results: Four patients with achalasia were found. All had Eckardt scores of ten or more and lower esophageal sphincter (LES) pressure of $30 \mathrm{~mm} \mathrm{Hg}$ or more. Surgical times ranged between 81 and 120 minutes. Follow-up examinations found that LES pressure had decreased by more than $50 \%$ in practically all patients. Eckardt scores were close to normal. This demonstrates success of POEM therapy after two months of evaluation. Complications were resolved intraoperatively without mortality. Conclusions: Management of achalasia by POEM has proven to be a safe and effective technique. Our first findings agree with the results described in the literature. This is the first step in the implementation of this novel approach for management of achalasia in our Colombian population.
\end{abstract}

Keywords

Achalasia, esophageal motility disorders, oral endoscopic myotomy, Heller myotomy. 


\section{INTRODUCCIÓN}

La miotomía endoscópica por vía oral (POEM) es una verdadera técnica quirúrgica realizada por un orificio natural (natural orifice transluminal endoscopic surgery -NOTES-) para el manejo de la acalasia, el cual es un trastorno crónico de la motilidad esofágica con aumento del tono del esfínter esofágico inferior (EEI), progresivo, y su tratamiento de elección es la sección de la musculatura circular, denominada miotomía de Heller, la cual se realiza en la actualidad por vía laparoscópica (MHL) con efectividad demostrada (1). Sin embargo, en 2007 el Dr. J. Pasricha y colaboradores (2)describieron el POEM en cerdos, sentando las bases de dicho procedimiento; el cual se llevó a cabo en humanos por primera vez en el 2008 por el Dr. Haruhiro Inoue, quien publicó su primera serie de casos en el 2010 (3). Es una técnica endoscópica que se encuentra en crecimiento, la cual ha demostrado resultados muy similares a la MHL, al evaluar la mejoría clínica (4); tiene como ventaja importante que, al ser un abordaje endoscópico puro, ofrece una recuperación rápida con estancias hospitalarias muy cortas y con una mortalidad de $0 \%$ reportada en una de las series más grandes con 500 pacientes (5).

Lo anterior lleva a considerar que este novedoso y prometedor abordaje endoscópico puede contribuir de manera positiva en la evolución y recuperación de los pacientes con acalasia. En este documento se publica la primera serie de casos en Colombia, realizados por un solo cirujano endoscopista y se analizan nuestros resultados.

\section{MATERIALES Y MÉTODOS}

Se realizó una revisión retrospectiva de las historias clínicas de los pacientes con diagnóstico de acalasia esofágica tratados con POEM en la Organización Clínica General del Norte, en Barranquilla, Colombia, entre junio del año 2015 y noviembre de 2017; esto procedimiento lo realizó un cirujano endoscopista con experiencia terapéutica. En todas las intervenciones se utilizó profilaxis antibiótica con cefazolina $1 \mathrm{~g}$ intravenosa (IV), dosis única, 30 minutos antes de la cirugía.

Equipos: Olympus Excera 2 CV-180 con unidad de dióxido de carbono $\left(\mathrm{CO}_{2}\right)$ y Fujinom VP-4450 HD con su unidad de $\mathrm{CO}_{2}$ Fujifilm GW100. Unidad ERBE VIO 200 S y VIO 300 D con unidad de hidrodisección ERBEJET 2.

\section{TÉCNICA QUIRÚRGICA}

- Paso 1, anestesia: paciente en decúbito dorsal bajo anestesia general e intubación nasotraqueal.

- Paso 2, lavado esofágico: se realiza con $500 \mathrm{cc}$ de solución salina normal (SSN) 0,9\% con endoscopio con canal terapéutico.
- Paso 3, identificación de los reparos anatómicos: cricofaríngeo, arco aórtico y bronquio fuente izquierdo, EEI y silueta de la columna vertebral, que a su vez se puede corroborar instilando $30 \mathrm{~mL}$ agua en la luz esofágica, observando el área de declive (Figura 1).

- Paso 4, pápula: inyección submucosa con $10 \mathrm{~mL}$ de dextrosa en agua destilada (DAD) $5 \%$ en SSN, mezclada con índigo carmín o azul de metileno junto con $80 \mathrm{mg}$ de gentamicina, inmediatamente inferior al reparo anatómico del arco aórtico y bronquio fuente izquierdo, en el horario de las 5 del reloj (Figura 2 A).

- Paso 5, mucosotomía: se realiza mucosotomía longitudinal de $1,5 \mathrm{~cm}$ con electrobisturí triangle knife o hybrid knife, incluyendo el tejido celular submucoso en el vértice más distal de la mucosotomía, lo que permite la introducción fácil del cup plástico distal del endoscopio. La aplicación de la mezcla del colorante se puede repetir durante la disección del tejido submucoso para facilitar la progresión en la disección del mismo (Figura 2 B).

- Paso 6, túnel submucoso: se fabrica el túnel submucoso con electrobisturí en modo spray coagulation, hasta sobrepasar $3 \mathrm{~cm}$ distal al cardias en el eje de las 5 y realizando la disección muy cercana a la capa muscular circular, la cual debe visualizarse totalmente horizontal (inferior) al eje del endoscopio y teniendo máximo cuidado de no lesionar la mucosa esofágica (Figura 2 C). Es de utilidad retirar el endoscopio en varias ocasiones del túnel submucoso, introduciéndolo en la luz esofágica para verificar que la disección se esté realizando en el eje de las 5 y para confirmar cuando la disección supere el EEI, lo cual se complementa observando el cardias en retroflexión y siguiendo la columna del índigo carmín.

- Paso 7, miotomía: se inicia $2 \mathrm{~cm}$ inferior a la mucosotomía, en el horario de las 5 del reloj y se continúa hasta $3 \mathrm{~cm}$ inferior al cardias, comprometiendo únicamente la capa muscular circular (interna) del esófago hasta observar las fibras musculares longitudinales (externa) (Figura 2 D).

- Paso 8, revisión y cierre de mucosotomía: revisión exhaustiva de hemostasia en el túnel, lavado de residuos con SSN y posterior succión suave, cierre de la mucosotomía con clips Olympus (azules), ubicando el primero $0,5 \mathrm{~cm}$ distal al vértice inferior de la mucosotomía, para permitir la formación de un pliegue que facilite la colocación del segundo clip y así sucesivamente hasta el cierre de la mucosotomía (6-8 clips). Se debe corroborar al final de la cirugía el cierre hermético de ambos lados de la hilera de clips (Figura 2 E).

Cuando finaliza el procedimiento, a todos los pacientes se les realiza una radiografía de tórax para descartar neumotórax, fundamentalmente. 24 horas después, se realiza un 


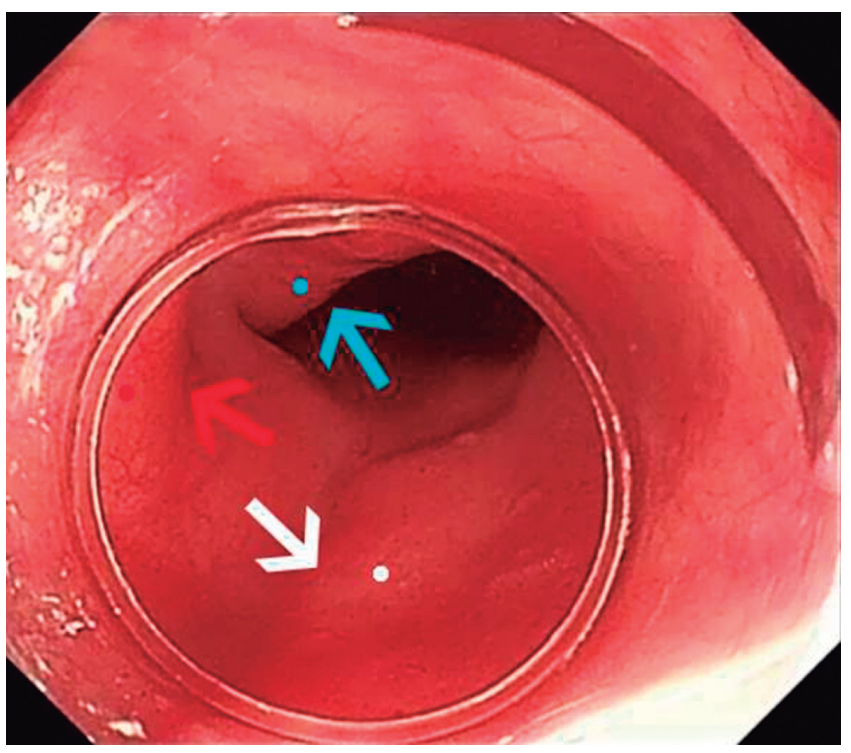

Figura 1. Reparos anatómicos: arco aórtico (flecha y punto rojos), bronquio fuente izquierdo (flecha y punto azules) y silueta de la columna vertebral (flecha y punto blancos) (6).

esofagograma con medio hidrosoluble; si no hay escapes, se inicia dieta líquida, que se mantiene por 1 semana y se avanza a blanda a la segunda semana, recuperando la dieta normal sobre la tercera semana.

\section{Seguimiento}

Se hizo seguimiento de los pacientes después de la cirugía al primer mes y se objetivizó a los 2 meses, en la consulta externa, con resultado de la manometría esofágica de alta resolución, puntaje Eckardt, esofagograma con bario y endoscopia de vías digestivas altas, esta última practicada por el endoscopista que realizó las cirugías.

\section{RESULTADOS}

Se encontraron 4 pacientes de género femenino con acalasia operadas por POEM entre junio de 2015 y noviembre de 2017, las características preoperatorias se especifican en la Tabla 1. Las pacientes tenían presión del EEI superiores a $30 \mathrm{~mm} \mathrm{Hg}$, una de ellas con Heller laparoscópico previo y 2 dilataciones neumáticas.

Los resultados postoperatorios se encuentran en la Tabla 2. Se encontró que 3 de 4 pacientes fueron operadas por vía posterior, debido a que se considera esta ruta más práctica al momento de realizar la miotomía, dado que la salida del electrobisturí se ubica en el eje de las 6 del equipo, quedando la cámara hacia las 12, lo que permite una mejor visualización del plano muscular a seccionar (Figura 3); solo el POEM 1 se realizó por vía anterior, hacia las 2 del reloj, lo que resultó en mayor tiempo y grado de dificultad en comparación con los otros 3 pacientes (POEM 2-4). En

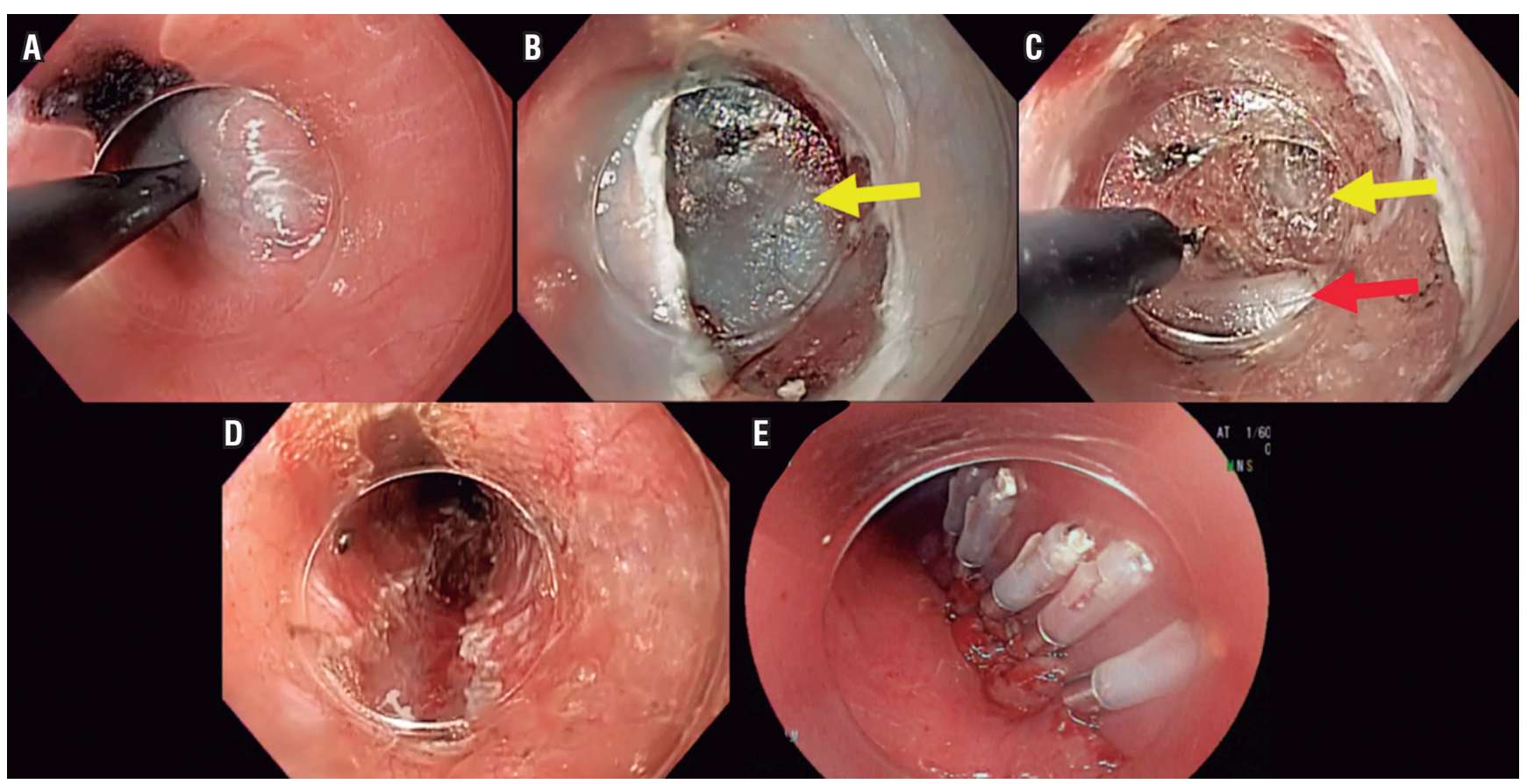

Figura 2. Pasos del POEM. A. Pápula. B. Mucosotomía (flecha amarilla: espacio submucoso). C. Túnel submucoso (flecha amarilla: espacio submucoso; flecha roja: capa muscular circular). D. Miotomía. E. Cierre de mucosotomía. 
Tabla 1. Características de los pacientes preoperatorias

\begin{tabular}{lccccccc}
\hline Variables & Edad (años) & Género & Puntaje ASA & Presión EEl & Clasificación Chicago & Puntaje Eckardt & Tratamientos previos \\
\hline POEM 1 & 32 & F & $\|$ & $38 \mathrm{~mm} \mathrm{Hg}$ & $\|$ & 10 & Dilatación \\
POEM 2 & 42 & F & $\|$ & $40 \mathrm{~mm} \mathrm{Hg}$ & $\|$ & 10 & No \\
POEM 3 & 52 & F & $\|$ & $35 \mathrm{~mm} \mathrm{Hg}$ & $\|$ & 12 & Dilatación \\
POEM 4 & 42 & F & $\|$ & $30 \mathrm{~mm} \mathrm{Hg}$ & $\|$ & 10 & Heller + Dor \\
& & & & & & Dilataciones (2) \\
\hline
\end{tabular}

ASA: American Society of Anesthesiologists.

Tabla 2. Resultados postoperatorios

\begin{tabular}{|c|c|c|c|c|}
\hline \multirow[t]{2}{*}{ Variables } & \multicolumn{4}{|c|}{ Pacientes } \\
\hline & POEM 1 & POEM 2 & POEM 3 & POEM 4 \\
\hline Duración & $120 \mathrm{~min}$ & $81 \mathrm{~min}$ & $80 \mathrm{~min}$ & $90 \min$ \\
\hline Longitud de miotomía total & $12 \mathrm{~cm}$ & $13 \mathrm{~cm}$ & $14 \mathrm{~cm}$ & $16 \mathrm{~cm}$ \\
\hline Longitud de miotomía del esófago & $10 \mathrm{~cm}$ & $11 \mathrm{~cm}$ & $11 \mathrm{~cm}$ & $13 \mathrm{~cm}$ \\
\hline Longitud de miotomía del estómago & $2 \mathrm{~cm}$ & $2 \mathrm{~cm}$ & $3 \mathrm{~cm}$ & $3 \mathrm{~cm}$ \\
\hline Vía de abordaje & Anterior & Posterior & Posterior & Posterior \\
\hline Complicaciones & $\begin{array}{c}\text { Enfisema subcutáneo } \\
\text { NP }\end{array}$ & $\begin{array}{c}\text { Enfisema subcutáneo } \\
\text { NP }\end{array}$ & $\begin{array}{c}\text { Enfisema subcutáneo } \\
\text { NP, NT }\end{array}$ & NP \\
\hline Presión EEI, control a los 2 meses & $19 \mathrm{~mm} \mathrm{Hg}$ & $18 \mathrm{~mm} \mathrm{Hg}$ & $12 \mathrm{~mm} \mathrm{Hg}$ & $16 \mathrm{~mm} \mathrm{Hg}$ \\
\hline Puntaje Eckardt, control a los 2 meses & 2 & 1 & 0 & 0 \\
\hline Esofagitis (clasificación Angeles) & A & A & 0 & 0 \\
\hline Estancia hospitalaria & 2 días & 1 día & 4 días & 1 día \\
\hline
\end{tabular}

NP: neumoperitoneo; NT: neumotórax.
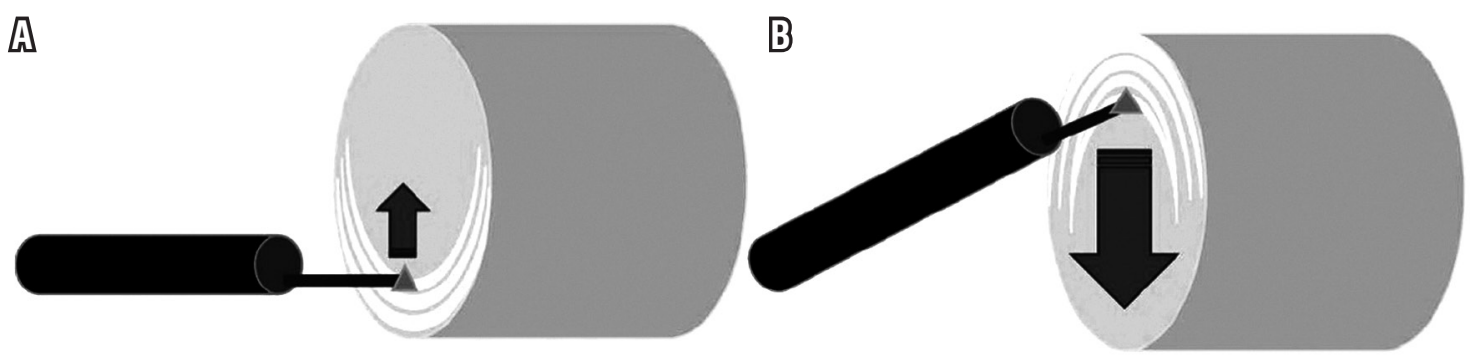

Figura 3. Vías para la realización de POEM (6). A. Posterior B. Anterior.

todos los pacientes se presentaron eventos derivados de la miotomía y la presión del $\mathrm{CO}_{2}$, el más común fue el capno/ neumoperitoneo, este evento se manejó durante el intraoperatorio con un yelco $14 \mathrm{G}$, localizado en el flanco derecho y conectado a una trampa de agua, sin complicaciones. Una de las pacientes presentó neumotórax izquierdo, el cual se manejó con un tubo de toracostomía $28 \mathrm{Fr}$ a sistema de drenaje cerrado y retirado 24 horas después, posterior a la realización de una radiografía de tórax que evidenciaba la resolución del neumotórax, es por esto que la paciente (POEM 3) tuvo una estancia hospitalaria mayor que el resto de pacientes, el enfisema subcutáneo no requiere manejo adicional, debido a que es autolimitado.

En nuestros casos se encontró una clara disminución de los síntomas derivados de la acalasia, lo cual se objetivó con el puntaje Eckardt, esto indica que la cirugía fue exitosa en el 100\% de los pacientes. Las manometrías esofágicas de alta resolución mostraron una evidente disminución de la 
presión del EEI, logrando prácticamente más del 50\% de reducción en comparación con el valor preoperatorio. La esofagitis se presentó en un grado leve y se manejó con inhibidores de bomba de protones (IBP), y de este modo se obtuvo una mejoría clínica más evidente y, por ende, una mejor calidad de vida para las pacientes. No hubo mortalidad alguna en esta serie de casos.

\section{DISCUSIÓN}

La acalasia es una enfermedad progresiva e irreversible médicamente, que puede ser idiopática en la mayoría de casos o secundaria (enfermedad de Chagas, virus del herpes, entre otras) $(1,7)$. Es más frecuente en personas entre la tercera y quinta década de la vida, como en nuestro grupo de pacientes. Su incidencia es de 0,4 al 1,2:100 000 habitantes y su prevalencia oscila entre 7 y 13:100 000 habitantes (8). El tratamiento puede ser farmacológico (7), toxina botulínica (9) con bajas tasas de éxito a largo plazo o con efectos secundarios que no son tolerados. Las guías de la American College of Gastroenterology (ACG) (7) y de la Society of American Gastrointestinal and Endoscopic Surgeons (SAGES) (10) recomiendan la dilatación neumática, con éxito del $93 \%$ a 4 años de seguimiento (11); sin embargo, con tiempo libre de disfagia desde $40 \%-78 \%$ a 5 años (12), y la MHL con un éxito en promedio de $89 \%$ (77\%-100\%) (13), incluso del $75 \%$ a 15,8 años de seguimiento (14).

Con la introducción en humanos del POEM en el año 2008 por el Dr. Inoue, se abre un nuevo horizonte en el manejo de esta patología, actualmente con más de 5000 procedimientos a nivel mundial, con buenos resultados, convirtiéndose en el primer NOTES exitoso $(3,15)$. Esta técnica quirúrgica también se ha empleado para trastornos motores de difícil manejo como el espasmo esofágico difuso y esófago de nutcracker/jackhammer $(16,17,18)$.

El éxito del POEM para el manejo de la acalasia se ha medido con 2 parámetros principalmente: la presión del EEI y el puntaje Eckardt (19), y se han encontrado tasas de éxito del $93 \%$, con muy baja incidencia de complicaciones mayores (20). Esta eficacia se ha demostrado en publicaciones como la de Haruhiro Inoue y colaboradores (5) de 500 pacientes con diagnóstico de acalasia llevados a POEM por vía anterior y posterior, en la que se evaluó a los pacientes con manometría esofágica de alta resolución y puntaje Eckardt a los 2 meses post-POEM. Se obtuvieron medidas pre y postoperatorias de $25,4 \pm 17,1$ frente a $13,4 \pm 5,9 \mathrm{~mm}$ $\mathrm{Hg}, \mathrm{p}<0,0001 ;$ y $6,0 \pm 3,0$ frente a $1,0 \pm 2,0, \mathrm{p}<0,0001$; respectivamente. Estos resultados dejaron ver una clara reducción de la presión del EEI y la sintomatología de los pacientes. Datos similares se obtuvieron en un metaanálisis realizado por Rupjyoti Talukdar y colaboradores, con 1045 pacientes en 19 estudios (21).
Nuestros resultados coinciden con la literatura mencionada, en los que se muestra una evidente mejoría del Eckardt y la presión del EEI, aunque se trata de una muestra pequeña. En la población estudiada hubo una paciente con MHL previo y posteriores dilataciones, lo cual le agregó al procedimiento un mayor grado de dificultad, debido al proceso fibrótico; sin embargo, esta es la circunstancia en la que tiene mayor utilidad la miotomía por vía posterior, debido a que el plano de disección se visualiza más claramente (Figura 4) $(5,22)$.

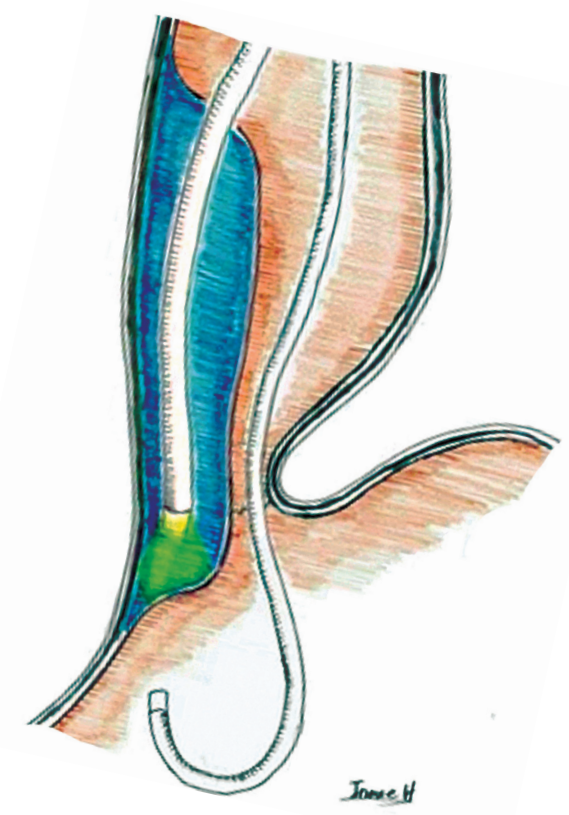

Figura 4. Técnica de doble endoscopio, se observa con el endoscopio ubicado en cámara gástrica; en retroflexión, la transiluminación del endoscopio que realiza el túnel submucoso y la miotomía (23).

Varios estudios han comparado los resultados del POEM con la MHL, uno de ellos es un metaanálisis publicado en 2015, que demostró riesgo de complicaciones (odds ratio [OR]: 1.17, intervalo de confianza [IC] 95\%: 0,53-2,56, $\mathrm{p}=0,70)$ y de reflujo gastroesofágico bajas (OR: 1,00 ; IC 95\%: 0,38-2,61; $\mathrm{p}=1,00)(24)$ compared with laparoscopic Heller's myotomy (LHM. Vivek Kumbhari y colaboradores publicaron en 2015 un estudio retrospectivo y multicéntrico en el que se comparó el POEM frente al MHL en pacientes con acalasia tipo III y se obtuvieron resultados superiores estadísticamente significativos a favor de la técnica endoscópica, en términos de mejoría clínica (98,0\% frente a $80,8 \% ; \mathrm{p}=0,01)$, menor tiempo operatorio (102 $\min$ frente a $264 \mathrm{~min} ; \mathrm{p}<0,01)$ y mayor longitud de miotomía ( $16 \mathrm{~cm}$ frente a $8 \mathrm{~cm}$; p <0,01) (4); este último factor permite el tratamiento con éxito en la acalasia tipo III, donde la miotomía debe ser de mayor longitud, lo cual no es posible mediante la MHL. 
En nuestros pacientes se obtuvo longitudes $\geq 12 \mathrm{~cm}$ con tiempos operatorios que cada vez fueron menores a medida que se aumentó el número de casos, incluso en los más complejos como la paciente del POEM 4.

La utilización del bisturí tipo hybrid knife ha demostrado una reducción significativa en tiempo quirúrgico y sangrado, en comparación con sistemas convencionales (triangle knife), esto debido a que brinda la posibilidad de realizar inyección para elevar el plano submucoso y al mismo tiempo como electrobisturí (25). Este dispositivo se utilizó en 2 pacientes y resultó ser más práctico porque disminuyó las veces de introducción y retiro de herramientas por el canal de trabajo del endoscopio.

Para los casos difíciles como, por ejemplo, pacientes con Heller, dilataciones y/o que han recibido toxina botulínica, que distorsionan la anatomía del EEI, puede utilizarse un segundo endoscopio, siendo este de diámetro pediátrico (Figura 3). En nuestros casos no hubo la necesidad de realizarlo, pero se puede lograr una longitud mayor de la miotomía gástrica con este método $(3,2 \mathrm{~cm}$ frente a $2,5 \mathrm{~cm} ; \mathrm{p}=$ $0,013)$ y menor índice de perforaciones a nivel de mucosa gástrica; sin embargo, no se obtiene un impacto significativo en el éxito de la técnica (23).

La esofagitis grado B/C puede presentarse hasta en el 16\% de los pacientes y es más frecuente cuando los valores de presión integrada de relajación (IRP) son bajos $(\approx 9,5 \mathrm{~mm}$ $\mathrm{Hg}$ ) en el control postoperatorio (26). En nuestros casos se observó que la mitad de los pacientes presentaron una esofagitis leve, los cuales respondieron a la terapia con IBP.

Las tasas de complicaciones son variables. K. Patel y colaboradores hicieron un análisis de 22 estudios con 1122 pacientes a quienes les realizó POEM, 392 pacientes tenían terapias previas para manejo de acalasia y reportaron las siguientes complicaciones: enfisema subcutáneo en 31,6\%, capno/neumoperitoneo en 30,6\%, capno/neumotórax en $11 \%$, capno/neumoperitoneo que requiere descompresión en $8 \%$, capno/neumotórax que requiere descompresión en $2,7 \%$, sangrado postoperatorio en $1,1 \%$, perforación esofágica/fuga mediastínica en $0,3 \%$ y una tasa de mortalidad del 0,09\%. Haruhiro Inoue y colaboradores, en la serie mencionada de 500 pacientes con acalasia manejados con POEM, 195 tenían tratamientos previos (10 Heller laparoscópico), obtuvo 3,2\% (16) de complicaciones (neumotórax con neumomediastino: 1 , sangrado: 1 , perforación de la mucosa: 8 , hematoma postoperatorio en el túnel: 3, inflamación en el omento menor: 1 y derrame pleural: 2 ) y una mortalidad de 0\% (5). Los hallazgos obtenidos se correlacionan con los observados en la literatura; a pesar de que todos nuestros pacientes presentaron neumoperitoneo, se pudo resolver con un yelco y una trampa de agua, sin resultar en una complicación mayor o mortalidad.
En los casos en que persiste la sintomatología posterior al primer POEM, se puede repetir el procedimiento en otro eje, con resultados seguros y con mejoría clínica importante. Así lo demostraron Amy Tyberg-Stefan Seewald y colaboradores en su estudio con 46 pacientes publicado en 2017 (27).

Con respecto a la curva de aprendizaje, esta se analizó en una serie prospectiva y se concluyó que para dominar la técnica se requieren por lo menos 40 casos de POEM, midiendo como variables el tiempo quirúrgico y el número de perforaciones en la mucosa; se observó que a partir de este número de casos se hacían más constantes estas 2 variables (28).

En conclusión, el POEM es una técnica efectiva y segura con base en la literatura actual, que la vía de miotomía posterior es más fácil técnicamente que la vía anterior, con baja morbilidad y que para su implementación no requiere equipos más complejos que los utilizados en una unidad de endoscopia terapéutica. Estos resultados están limitados por el pequeño tamaño de muestra $y$, por ende, no se pueden emitir juicios con alto poder estadístico; sin embargo, son el primer paso para la implementación y ampliación del uso de POEM en nuestra población.

\section{Agradecimientos}

El presente trabajo fue realizado gracias a la colaboración y apoyo de la institución Clínica General del Norte en Barranquilla, Colombia, que contribuyó con su personal humano, instalaciones, equipo y apoyo en la realización de este trabajo.

\section{REFERENCIAS}

1. Pandolfino JE, Gawron AJ. Achalasia: a systematic review. JAMA. 2015;313(18):1841-52. doi: 10.1001/ jama.2015.2996.

2. Pasricha PJ, Hawari R, Ahmed I, Chen J, Cotton PB, Hawes $\mathrm{RH}$, et al. Submucosal endoscopic esophageal myotomy: a novel experimental approach for the treatment of achalasia. Endoscopy. 2007;39(9):761-4. doi: 10.1055/s-2007-966764.

3. Inoue H, Minami H, Kobayashi Y, Sato Y, Kaga M, Suzuki M, et al. Peroral endoscopic myotomy (POEM) for esophageal achalasia. Endoscopy. 2010;42(4):265-71. doi: 10.1055/s0029-1244080.

4. Kumbhari V, Tieu A, Onimaru M, El Zein MH, Modayil RJ, Teitelbaum EN, et al. 730 PerOral Endoscopic Myotomy (POEM) Versus Laparoscopic Heller Myotomy (Lhm) for the Treatment of Type III Achalasia in 75 Patients: an International Multicenter Experience. Gastrointest Endosc. 2014;79(5):AB166. doi: 10.1016/j.gie.2014.02.167.

5. Inoue H, Sato H, Ikeda H, Onimaru M, Sato C, Minami H, et al. Per-Oral Endoscopic Myotomy: A Series of 500 Patients. J Am Coll Surg. 2015;221(2):256-64. doi: 10.1016/j.jamcollsurg.2015.03.057. 
6. Bechara R, Onimaru M, Ikeda H, Inoue H. Per-oral endoscopic myotomy, 1000 cases later: pearls, pitfalls, and practical considerations. Gastrointest Endosc. 2016;84(2):330-8. doi: 10.1016/j.gie.2016.03.1469.

7. Vaezi MF, Pandolfino JE, Vela MF. ACG clinical guideline: diagnosis and management of achalasia. Am J Gastroenterol. 2013 Aug;108(8):1238-49. doi: 10.1038/ajg.2013.196.

8. O'Connor JB, Singer ME, Imperiale TF, Vaezi MF, Richter JE. The cost-effectiveness of treatment strategies for achalasia. Dig Dis Sci. 2002;47(7):1516-25.

9. Martínek J, Siroký M, Plottová Z, Bures J, Hep A, Spicák J. Treatment of patients with achalasia with botulinum toxin: a multicenter prospective cohort study. Dis Esophagus. 2003;16(3):204-9.

10. Stefanidis D, Richardson W, Farrell TM, Kohn GP, Augenstein V, Fanelli RD, et al. SAGES guidelines for the surgical treatment of esophageal achalasia. Surg Endosc. 2012;26(2):296-311. doi: 10.1007/s00464-011-2017-2.

11. Kadakia SC, Wong RK. Pneumatic balloon dilation for esophageal achalasia. Gastrointest Endosc Clin N Am. 2001;11(2):325-46, vii.

12. Zerbib F, Thétiot V, Richy F, Benajah DA, Message L, Lamouliatte H. Repeated pneumatic dilations as longterm maintenance therapy for esophageal achalasia. Am J Gastroenterol. 2006;101(4):692-7. doi: 10.1111/j.15720241.2006.00385.x.

13. Campos GM, Vittinghoff E, Rabl C, Takata M, Gadenstätter M, Lin F, et al. Endoscopic and surgical treatments for achalasia: a systematic review and metaanalysis. Ann Surg. 2009;249(1):45-57. doi: 10.1097/ SLA.0b013e31818e43ab.

14. Csendes A, Braghetto I, Burdiles P, Korn O, Csendes P, Henríquez A. Very late results of esophagomyotomy for patients with achalasia: clinical, endoscopic, histologic, manometric, and acid reflux studies in 67 patients for a mean follow-up of 190 months. Ann Surg. 2006;243(2):196-203. doi: 10.1097/01.sla.0000197469.12632.e0.

15. Mejía R, Donoso A, Manríquez E, Sáez J, Crovari F, Gabrielli $\mathrm{M}$, et al. Miotomía endoscópica por vía oral (POEM) para el tratamiento de la acalasia. Experiencia clínica inicial y resultados a corto plazo. Rev Chil Cirugía. 2017;69(5):429-34. doi: 10.1016/j.rchic.2017.03.004.

16. Khashab MA, Messallam AA, Onimaru M, Teitelbaum EN, Ujiki MB, Gitelis ME, et al. International multicenter experience with peroral endoscopic myotomy for the treatment of spastic esophageal disorders refractory to medical therapy (with video). Gastrointest Endosc. 2015 May;81(5):11707. doi: 10.1016/j.gie.2014.10.011.

17. Paterson WG, Beck IT, Da Costa LR. Transition from nutcracker esophagus to achalasia. A case report. J Clin Gastroenterol. 1991;13(5):554-8.
18. Khan MA, Kumbhari V, Ngamruengphong S, Ismail A, Chen YI, Chavez YH, et al. Is POEM the Answer for Management of Spastic Esophageal Disorders? A Systematic Review and Meta-Analysis. Dig Dis Sci. 2017;62(1):35-44. doi: 10.1007/s10620-016-4373-1.

19. Eckardt AJ, Eckardt VF. Treatment and surveillance strategies in achalasia: an update. Nat Rev Gastroenterol Hepatol. 2011;8(6):311-9. doi: 10.1038/nrgastro.2011.68.

20. Barbieri LA, Hassan C, Rosati R, Romario UF, Correale L, Repici A. Systematic review and meta-analysis: Efficacy and safety ofPOEM for achalasia. UnitedEuropean Gastroenterol J. 2015;3(4):325-34. doi: 10.1177/2050640615581732.

21. Talukdar R, Inoue H, Nageshwar Reddy D. Efficacy of peroral endoscopic myotomy (POEM) in the treatment of achalasia: a systematic review and meta-analysis. Surg Endosc. 2015;29(11):3030-46. doi: 10.1007/s00464-014-4040-6.

22. Nabi Z, Ramchandani M, Chavan R, Kalapala R, Darisetty $S$, Rao GV, et al. Per-oral endoscopic myotomy for achalasia cardia: outcomes in over 400 consecutive patients. Endosc Int Open. 2017;5(5):E331-E339. doi: 10.1055/s-0043-105517.

23. Grimes $\mathrm{KL}$, Inoue $\mathrm{H}$, Onimaru $\mathrm{M}$, Ikeda $\mathrm{H}$, Tansawet $\mathrm{A}$, Bechara $\mathrm{R}$, et al. Double-scope per oral endoscopic myotomy (POEM): a prospective randomized controlled trial. Surg Endosc. 2016;30(4):1344-51. doi: 10.1007/s00464015-4396-2.

24. Wei M, Yang T, Yang X, Wang Z, Zhou Z. Peroral esophageal myotomy versus laparoscopic Heller's myotomy for achalasia: a meta-analysis. J Laparoendosc Adv Surg Tech A. 2015;25(2):123-9. doi: 10.1089/lap.2014.0454.

25. Cai MY, Zhou PH, Yao LQ Xu MD, Zhong YS, Li QL, et al. Peroral endoscopic myotomy for idiopathic achalasia: randomized comparison of water-jet assisted versus conventional dissection technique. Surg Endosc. 2014;28(4):115865. doi: 10.1007/s00464-013-3300-1.

26. Shiwaku H, Inoue $H$, Sasaki T, Yamashita $K$, Ohmiya T, Takeno S, et al. A prospective analysis of GERD after POEM on anterior myotomy. Surg Endosc. 2016;30(6):2496-504. doi: $10.1007 /$ s00464-015-4507-0.

27. Tyberg A, Seewald S, Sharaiha RZ, Martinez G, Desai AP, Kumta NA, et al. A multicenter international registry of redo per-oral endoscopic myotomy (POEM) after failed POEM. Gastrointest Endosc. 2017;85(6):1208-1211. doi: 10.1016/j.gie.2016.10.015.

28. Kurian AA, Dunst CM, Sharata A, Bhayani NH, Reavis KM, Swanström LL. Peroral endoscopic esophageal myotomy: defining the learning curve. Gastrointest Endosc. 2013;77(5):719-25. doi: 10.1016/j.gie.2012.12.006. 